\title{
Shift Metrics - Software de coleta de medidas e análise de indicadores com aderência aos requisitos exigidos pelo MPS.BR, desenvolvido e utilizado por uma empresa certificada MPS.BR Nível C
}

\author{
Gustavo V. Nascimento ${ }^{1}$, Thaynan M. Antunes ${ }^{1}$, Edilson C. Junior ${ }^{1}$, Leonardo F. \\ Oliveira $^{1,}$ Francielli C. André ${ }^{1}$ \\ ${ }^{1}$ Shift Informática para Laboratórios \\ Rua Independência, 3281 CEP 15.010-110 - São José do Rio Preto - SP - Brasil \\ \{gustavo, thaynan, junior, leonardo, francielli\} eshift.com.br
}

\begin{abstract}
The measurement process is essential for organizations to achieve their improvement goals because it allows to understand how their processes and projects behave. However, as it has been pointed in the literature, the low quality of data and metrics affects the improvement process. This paper presents Shift Metrics - a measurement software - and the strategy adopted by Shift - a laboratory software development company certified MPS.Br Level C - to develop it. Shift Metrics objectives are to address the organization needs and MPS.Br requirements and to provide a well-structured metrics database that can be successfully used in high level maturity processes of MPS.Br.
\end{abstract}

Resumo. O processo de medição é fundamental para que as organizações alcancem seus objetivos de melhoria, pois ele permite o entendimento do comportamento de seus processos e projetos. Entretanto, tem sido destacado na literatura, que a baixa qualidade referente à coleta de dados e medidas interfere negativamente no processo de melhoria. Este artigo apresenta o Shift Metrics e a estratégia adotada pela Shift - empresa de desenvolvimento de software para laboratório de análises clínicas certificada MPS.Br Nivel C para desenvolve-lo. O Shift Metrics é um software de apoio ao processo de medição que visa atender as necessidades intrínsecas à organização, os requisitos do MPS.Br e preparar a base de medidas da organização para atender aos requisitos exigidos pelos altos níveis de maturidade do MPS.Br.

\section{Introdução}

A evolução tecnológica e a alta competitividade no mercado de software aumentam continuamente a demanda por softwares cada vez melhores e mais aderentes aos custos, prazos e qualidades desejados. Em paralelo, as organizações têm aumentado significativamente o interesse pela melhoria de processos de software. Muitas delas recorrem a modelos de melhoria de processos como o MPS.Br, CMMI-Dev, ISO/IEC 12207 e ISO/IEC 15504. Nesses modelos o processo de medição ocupa um papel fundamental [Barcellos-c 2010, ISO/IEC-12207 2008, ISO/IEC-15504 2003, CMMI 2010].

No modelo MPS.Br, o processo de medição é introduzido no Nível F. Nos modelos citados, o processo de medição tem como propósito coletar, armazenar, 
analisar e relatar dados relacionados aos processos implementados e produtos desenvolvidos pela organização, apoiando os processos de gerência e melhoria de processo e de produto [SOFTEX-a 2009]. O principal foco da medição é fazer com que a organização entenda como os projetos e processos atendem aos objetivos organizacionais, provendo informação útil para a tomada de decisões que impactam seus objetivos de negócio. Embora o processo de medição seja inserido na organização quando esta está almejando a maturidade de Nível F, com o aumento de maturidade da organização, as medições tornam-se indispensáveis para o dia a dia. Com isso, faz-se necessário o controle e armazenamento adequado das medidas, indicadores e informações relacionadas, para que seja possível um entendimento adequado do contexto em que os dados foram coletados e, consequentemente, das decisões que precisam ser tomadas. A qualidade dos dados armazenados neste contexto também é um fator relevante para o sucesso do processo, pois aumenta a confiabilidade durante o processo de análise e tomada de decisão. A literatura indica que empresas que almejam altos níveis de maturidade tem enfrentado dificuldades para implantar controle estatístico de processo, por terem dados e medidas coletadas inadequadamente [Barcellos-c 2010].

Com isso, o Shift Metrics foi desenvolvido pela Shift Informática para Laboratórios - empresa de desenvolvimento de software para laboratório de análises clínicas, com 22 anos de existência e certificada MPS.Br Nível C - com o objetivo de atender aos requisitos exigidos pelo processo de medição do MPS.Br; de armazenar as medidas, indicadores e informações relacionadas de forma que permita a recuperação destas dentro do contexto em que foram coletadas; de atender a necessidade de evolução do processo de medição dentro da organização; de propiciar confiabilidade aos dados coletados; e de preparar a base de medidas para o controle estatístico de processo, caracterizado nos níveis A e B do MPS.Br.

Este artigo está organizado da seguinte forma: Na Seção 2 estão apresentados os itens que originaram o projeto Shift Metrics e os principais resultados almejados. $\mathrm{Na}$ Seção 3 o Shift Metrics é descrito em conjunto com o processo de medição da organização. $\mathrm{Na}$ Seção 4 são apresentadas as considerações finais a respeito da experiência vivida com a construção e evolução do Shift Metrics. Finalmente, na Seção 5 são apresentadas as referências citadas neste artigo.

\section{O projeto Shift Metrics}

O alcance da alta maturidade, caracterizada nos níveis A e B do MPS.Br, requer a implementação do controle estatístico de processos, que somente pode ser realizado se forem utilizados medidas e dados adequados a esse contexto. Muitas organizações que iniciam as práticas necessárias à alta maturidade precisam adiar a implementação do controle estatístico de processos devido à inadequação de suas bases de medidas [Barcellos-c 2010].

Este cenário, em conjunto com a necessidade da organização em ter uma ferramenta que apoiasse seu processo de medição e que se adequasse às frequentes evoluções dos conjuntos de medidas e indicadores (comum nas fases iniciais de implantação do processo de medição), fez com que a organização decidisse (em 2010) iniciar o desenvolvimento do Shift Metrics. Para que o software atendesse as expectativas da organização, foram definidos os seguintes objetivos: 
1. Atender aos requisitos exigidos pelo processo de medição do MPS.Br.

2. Armazenar as medidas, indicadores, análises e informações relacionadas de forma que fosse possível a recuperação destas dentro do contexto em que foram coletadas, auxiliando, desta forma, as análises periódicas previstas na organização.

3. Atender a necessidade de evolução do processo de medição e do conjunto de medidas e indicadores da organização.

4. Propiciar confiabilidade aos dados coletados.

5. Preparar a base de medidas para o controle estatístico de processo, caracterizado nos níveis A e B do MPS.Br.

Os objetivos de 1 a 4 foram definidos com base em pesquisas sobre relatos de experiência em implementações do processo de medição em outras organizações, na experiência da própria Shift na execução deste processo e na expectativa da organização em ter dados confiáveis para a análise e tomada de decisões. O Objetivo 5 foi definido em virtude do planejamento estratégico da organização, que almeja atingir os níveis A e $\mathrm{B}$ do MPS.Br.

\section{O processo de medição da Shift e o software Shift Metrics}

O processo de medição da Shift tem como ponto central o Plano de Medição. Ele é a base para todas as medições realizadas na organização que se referem aos projetos ou processos que estão dentro do escopo do MPS.Br e contém todas as informações necessárias para a realização das coletas e análises.

Com este contexto e importância, é crucial que a publicação de alterações no Plano de Medição passe por auditorias de qualidade. Na Shift, elas ocorrem em duas etapas. A primeira ocorre após as alterações e antes da publicação do plano, visando prevenir inconsistências frente aos objetivos organizacionais. A segunda ocorre após a transferência das alterações para o Shift Metrics e antes das alterações entrarem em vigor, visando prevenir erros no processo de transferência. Estas exigências visam atender ao Objetivo 4 relatado na Seção 2.

O Plano de Medição define as medidas e indicadores que devem ser coletados, vincula-os aos objetivos estratégicos da organização, define como e quando os dados devem ser coletados, quem deve coletar, interpretar e analisar cada medida e indicador e onde os resultados devem ser registrados. Na Figura 1 é apresentada a estrutura do plano de medição, incluindo os relacionamentos existentes entre cada item. A estrutura foi replicada para o Shift Metrics, tornando-o uma reprodução fiel das definições existentes.

Os itens presentes na estrutura do Plano de Medição são descritos a seguir:

- Medida ${ }^{1}$ (básica ou derivada) e informações necessárias relacionadas (orientações de coleta, responsável pela coleta, descrição, localização dos dados, outros).

\footnotetext{
${ }^{1}$ Medida básica é aquela definida em termos de um único atributo por método de medição. Medida derivada é aquela definida em função de dois ou mais valores de medidas básicas ou derivadas. [SOFTEX-b 2009]
} 
- Indicador ${ }^{2}$ e informações necessárias relacionadas (medidas relacionadas, fórmula para cálculos, faixa de valores aceitável ou desejável pela organização, orientações para interpretação e análise, responsável pela interpretação e análise, responsável pela aprovação e análise, outros).

- Forma de visualização gráfica (tipo de gráfico que será utilizado para exibir o indicador - gráfico de pizza, gráfico de linhas, gráfico de barras).

- Ponto de medição (momento no tempo onde um indicador ou conjunto de indicadores devem ser interpretados e analisados e os resultados devem ser registrados).

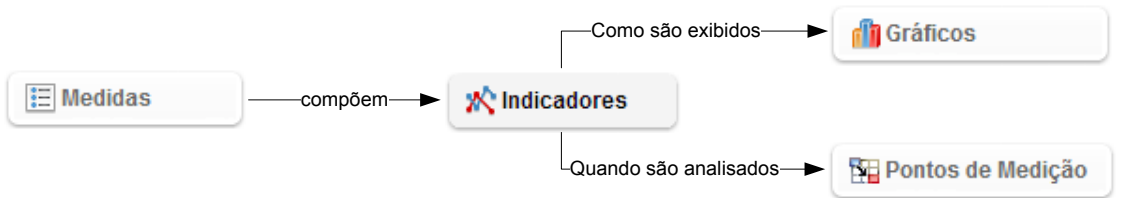

Figura 1: Estrutura de definição do Plano de Medição (a estrutura foi replicada para o Shift Metrics)

O Plano de Medição evolui constantemente e tais evoluções normalmente afetam os relacionamentos entre medidas, indicadores, gráficos e pontos de medição e podem impactar o monitoramento de projetos que estão em andamento. Considerando que as evoluções ocorrem ao longo do tempo, é preciso haver mecanismos que auxiliem a organização a recuperar o contexto em que as medições ocorreram. O contexto é importante, principalmente, em análises futuras, quando os responsáveis pelas medições não se lembrarem do cenário em que as medições foram realizadas ou quando não estiverem mais na organização.

Com isso, era importante que o Shift Metrics contivesse recursos que atendessem a essas necessidades. Além disso, durante o período de evolução do Shift Metrics e do próprio processo de medição, a organização encontrou alguns questionamentos que precisaram ser respondidos. Para ilustrar tais questionamentos, na Tabela 1 é apresentada uma situação comum em uma empresa certificada Nível C do MPS.Br (os exemplos utilizados referem-se a indicadores reais da organização):

Tabela 1: Situação comum em uma empresa certificada Nível C do MPS.Br

Durante análises do plano de medição em busca de oportunidades de melhoria, a organização decide que:

1. A periodicidade de análise do indicador EDD (Eficácia de Deteç̧ão de Defeitos) deve passar a ser mensal (não trimestral como era anteriormente).

2. A orientação de interpretação do indicador PDITS (Proporção de Defeitos Internos de Testes de Sistema) deve ser alterada.

3. A faixa aceitável para desvio de prazos dos projetos (estimado x realizado) deve passar a ser de $5 \%$ para mais ou para menos (não $10 \%$ como era anteriormente).

\footnotetext{
${ }^{2}$ Um indicador é uma estimativa ou avaliação que provê uma base para a tomada de decisão [SOFTEX-b 2009].
} 
A situação descrita relata uma necessidade clara de evolução. Como tratar esta necessidade? A seguir são apresentados alguns questionamentos importantes:

a. Uma nova versão do Plano de Medição precisa ser gerada para contemplar as alterações?

b. Os projetos em andamento serão impactados? Quais deles precisam dessas alterações para que o monitoramento torne-se efetivo?

c. Para os projetos que precisam das alterações: $\mathrm{O}$ que acontecerá com as medidas, indicadores e análises realizadas seguindo a versão anterior do Plano de Medição? A nova orientação de interpretação (item 2 da Tabela 1) tornará inválidas as análises previamente realizadas?

d. Durante uma provável análise futura dos dados armazenados, a organização se lembrará de que houve uma alteração da versão do Plano de Medição? O contexto/cenário em que as medições foram realizadas será importante?

Para responder esses questionamentos, a organização utilizou-se da experiência com a execução do processo adquirida até então e, principalmente, recorreu à literatura relacionada ao assunto. O trabalho intitulado "IABM: Instrumento para Avaliação de Bases de Medidas para Controle Estatístico de Processos de Software" [Barcellos-a 2010] foi a principal fonte de referência para a definição da estrutura da base de dados de medidas, visto que o trabalho define requisitos essenciais para o armazenamento de informações de contexto (previamente identificado neste artigo como importante para a Shift) e para a preparação da base de dados de medidas para o controle estatístico de processo (ressaltando que a preparação da base de dados de medidas para os altos níveis de maturidade era um dos objetivos do projeto de construção do Shift Metrics, conforme apresentado na Seção 2).

As respostas obtidas foram definidas como requisitos para o Shift Metrics, que possui um controle de versões de Planos de Medição e permite que qualquer projeto (em andamento ou não) seja vinculado a uma versão específica do plano. Desta forma, considerando a situação descrita e após as devidas análises referentes aos questionamentos "a" e "b", o projeto pode ser vinculado à nova versão do plano. A partir deste vínculo, todas as coletas do projeto passam a respeitar as definições da nova versão do plano.

Quando um novo vínculo entre projeto e versão do plano de medição é criado, todas as informações coletadas previamente no projeto continuam vinculadas à versão do plano de medição utilizada para aquela coleta. Isso significa que, em futuras análises de dados, o contexto definido naquela versão do plano permanece acessível durante as interpretações e análises.

Toda coleta de medidas respeita a estrutura definida no Plano de Medição, portanto, a coleta é feita para um determinado projeto e em um determinado ponto de medição. Durante a coleta, todas as informações necessárias para a realização adequada da coleta são apresentadas (Figura 2), minimizando falhas por falta de conhecimento sobre a forma de coleta e aumentando a qualidade dos dados coletados.

Após a coleta de todas as medidas referentes a um ponto de medição, os indicadores podem ser calculados e, posteriormente, interpretados e analisados pelos responsáveis. 


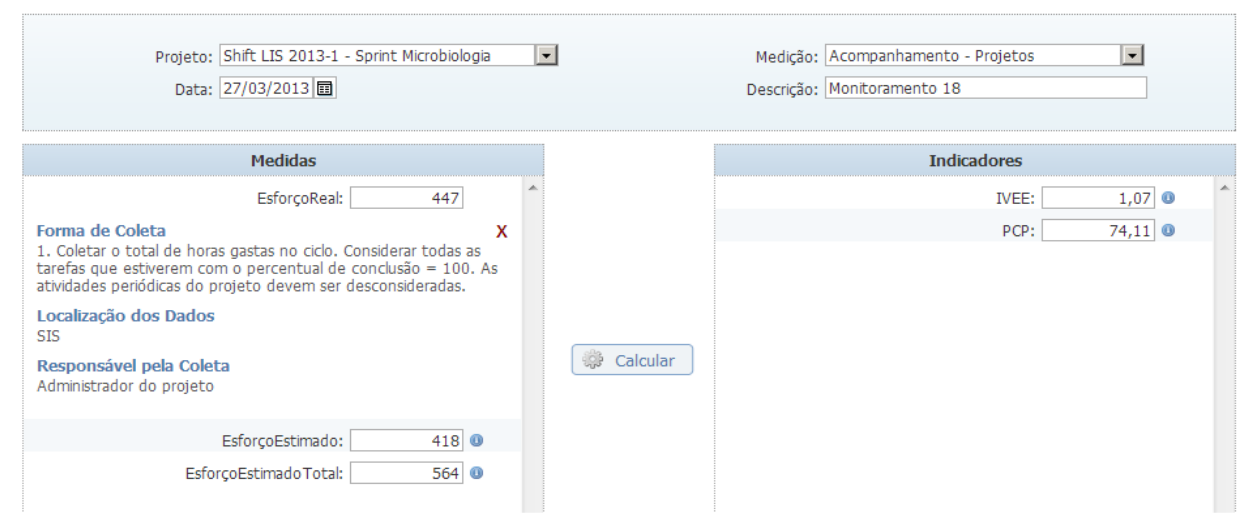

Figura 2: Funcionalidade de coleta de medidas e cálculo de indicadores do projeto "Shift LIS 2013-1 - Sprint Microbiologia", ponto de medição "Acompanhamento - Projetos"

Durante a atividade de interpretação e análise, o responsável pode visualizar, dentre outras informações: os registros de análises anteriores, os dados das medidas que compõem o indicador de forma isolada e o responsável pela coleta de cada medida. Este recurso propicia uma visão bastante realista do que está acontecendo no projeto ou processo em análise e permite uma melhor interpretação dos dados.

Após o registro da análise, a atividade ainda passa por uma aprovação, cujo responsável é definido no Plano de Medição. A aprovação e o responsável por ela são registrados no Shift Metrics e somente depois disso os indicadores são divulgados aos interessados (a divulgação ocorre de forma automática por e-mail). Este foi o mecanismo que a Shift encontrou para garantir que as orientações de interpretação e análise sejam seguidas e, com isso, minimizar problemas de confiabilidade dos dados. $\mathrm{Na}$ Figura 3 e na Figura 4 está apresentada a tela de registro das interpretações e análises, mostrando, respectivamente, as informações disponíveis para a realização da análise (em 27/03/2013) e as informações de análises anteriores (as informações apresentadas são referentes às análises realizadas em 14/11/2012).

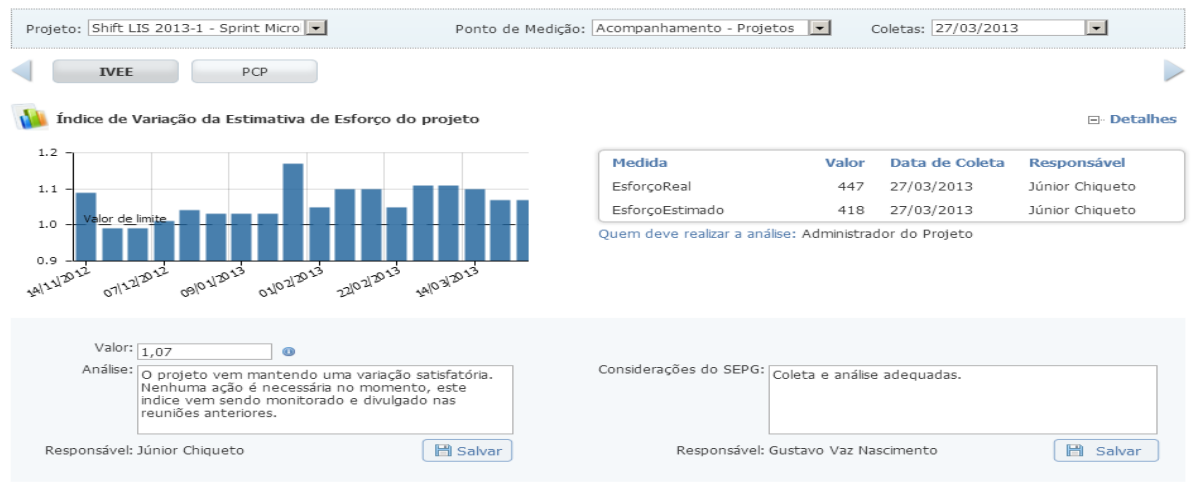

Figura 3: Funcionalidade de registro de interpretação e análise de indicadores

O Shift Metrics possui ainda a possibilidade de consolidar indicadores coletados em projetos diferentes, exibindo e registrando o resultado da consolidação em um gráfico (os indicadores a serem consolidados e o tipo de gráfico a ser exibido é definido no Plano de Medição). Este recurso é útil, por exemplo, quando se deseja ter uma visão 
de como estão os indicadores de prazo (estimado x realizado) de toda a organização, consolidando os projetos em andamento ou finalizados até uma determinada data (a organização seleciona quais projetos deseja consolidar). Nesta situação, é possível selecionar os projetos e o indicador que se deseja consolidar, assim, o Shift Metrics apresenta um gráfico com o resultado da consolidação e as análises podem prosseguir como apresentado anteriormente. Na Figura 5 está apresentada a funcionalidade de consolidação com a lista de projetos selecionados para ela.

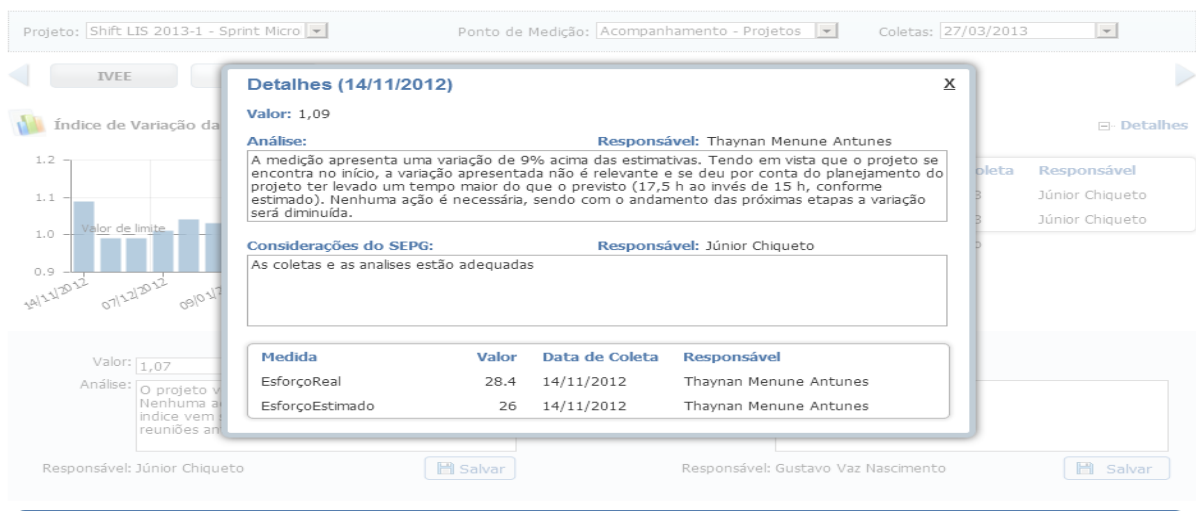

Figura 4: Funcionalidade de registro de interpretação e análise de indicadores com exibição de detalhes de um ponto anterior específico no tempo

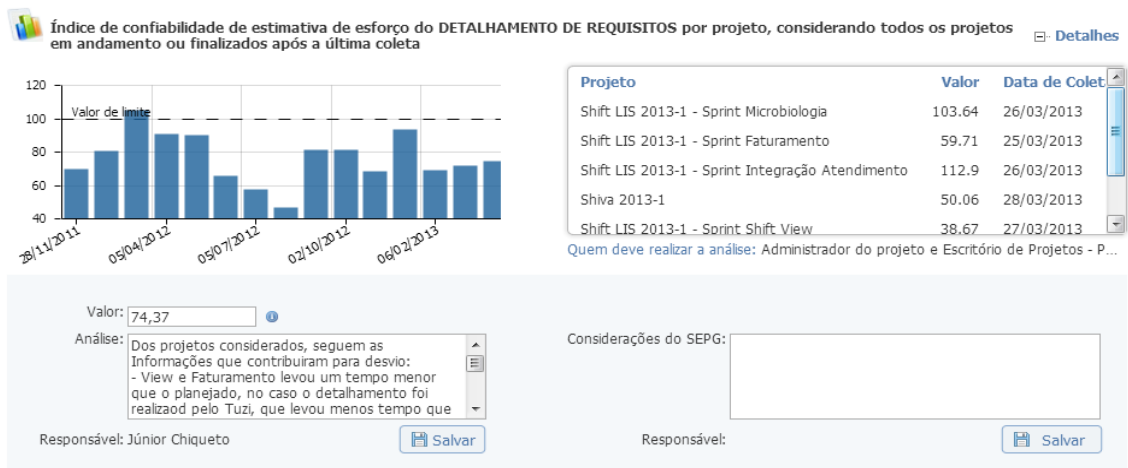

Figura 5: Funcionalidade de análise de indicadores consolidados com a lista de projetos e respectivos valores individuais do indicador selecionado.

\section{Considerações finais}

Os cinco objetivos definidos para o Shift Metrics, listados na Seção 2 deste artigo, foram parcialmente atendidos, visto que a Shift ainda não é uma empresa certificada Nível B e, portanto, ainda não é possível afirmar que a base de dados de medidas está adequada ao controle estatístico de processos (Objetivo 5). No entanto, a adequação aos processos do Nível B torna-se foco principal de futuros trabalhos em direção a melhoria de processo.

Com relação ao Objetivo 1 (atender aos requisitos do processo de medição do MPS.Br), considera-se totalmente satisfeito, pois o Shift Metrics foi objeto da auditoria que indicou a Shift para o Nível C de Maturidade.

O Objetivo 2 (recuperar informações do contexto da coleta) e o Objetivo 3 (evoluções do processo e do conjunto de medidas e indicadores) também foram 
considerados como satisfeitos. Com relação ao Objetivo 2, alguns recursos do Shift Metrics possibilitam a recuperação de contextos de coleta: 1 . Contexto referente à versão do plano de medição utilizada no momento da coleta, permitindo a recuperação de todas as definições relacionadas àquela versão do plano; 2 . Contexto referente ao projeto, permitindo a recuperação de coletas e análises anteriores, de detalhes das medidas que compõem os indicadores, de responsáveis pelas coletas e análises e dos pontos de medição. Os mesmos recursos podem ser utilizados para evidenciar o atendimento ao Objetivo 3, pois eles permitem observar que as evoluções do processo são tratadas pelo Shift Metrics de forma bastante satisfatória.

O Objetivo 4 (propiciar a confiabilidade dos dados) é atendido através dos mecanismos de auditoria de qualidade sobre as alterações do Plano de Medição e sobre a transferências das alterações do plano para o Shift Metrics (a auditoria é registrada no próprio Shift Metrics). Outro mecanismo importante que tem relação com este objetivo é a necessidade de aprovação das análises antes da publicação. Estes mecanismos aumentam significativamente a confiança nos dados armazenados.

Com tudo o que foi apresentado, é possível concluir que a experiência e o aprendizado adquiridos durante a utilização do processo de medição, as pesquisas realizadas em busca do conhecimento necessário para conduzir o projeto Shift Metrics e o alinhamento do projeto com as diretrizes estratégicas da organização foram fundamentais para os resultados alcançados.

\section{Referências}

[Barcellos-a 2010] Barcellos, M. P., Rocha, A. R., \& Falbo, R. A. (2010). IABM: Instrumento para Avaliação de Bases de Medidas para Controle Estatístico de Processos de Software. IX Simpósio Brasileiro de Qualidade de Software, 119133. Belém, PA, Brasil.

[Barcellos-c 2010] Barcellos, M. P., Rocha, A. R., \& Santos, G. (2010). Análise da Estrutura e Conteúdo de uma Base de Medidas. IX Simpósio Brasileiro de Qualidade de Software, 311-318. Belém, PA, Brasil.

[SOFTEX-a 2009] Sociedade SOFTEX - Guia Geral. (05 de 2009). MPS.Br - Melhoria de Processo do Software Brasileiro: Guia Geral. Guia Geral. Acesso em 28 de 03 de 2013, disponível em http://www.softex.br/mpsbr

[SOFTEX-b 2009] Sociedade SOFTEX - Parte 2. (05 de 2009). MPS.BR - Melhoria de Processo do Software Brasileiro: Guia de Implementação - Parte 2: Fundamentação para Implementação do Nível F do MR-MPS.

[ISO/IEC-12207 2008] ISO/IEC (2008) "ISO/IEC 12207:2008 - Systems and Software Engineering - Software Life Cycle Process", International Organization for Standardization and the International Electrotechnical Commission, Switzerland.

[ISO/IEC-15504 2003] ISO/IEC (2003) "ISO/IEC 15504-2 - Information Technology Software Process Assessment", International Organization for Standardization and the International Electrotechnical Commission, Geneva, Switzerland.

[CMMI 2010] Carnegie Mellon Software Engineering Institute (08 de 2010). "CMMI ${ }^{\circledR}$ para Desenvolvimento - Versão 1.3: Melhoria de processos visando melhores produtos", Pittsburgh, Pennsylvania, United States. 